\title{
Highly Compact Robots for Inspection of Power Plants
}

\section{Conference Paper}

\section{Author(s):}

Caprari, Gilles; Breitenmoser, Andreas; Fischer, Wolfgang; Hürzeler, Christoph; Tâche, Fabien; Siegwart, Roland; Schoeneich, Patrick; Rochat, Frédéric; Mondada, Francesco; Moser, Roland

\section{Publication date:}

2010

Permanent link:

https://doi.org/10.3929/ethz-a-010034956

\section{Rights / license:}

In Copyright - Non-Commercial Use Permitted 


\title{
Highly Compact Robots for Inspection of Power Plants
}

\author{
Gilles Caprari, Andreas Breitenmoser, Wolfgang Fischer, Christoph Hürzeler, Fabien Tâche, Roland \\ Siegwart, Patrick Schoeneich, Frédéric Rochat, Francesco Mondada, Roland Moser
}

\begin{abstract}
This paper reports on a 3 years applied research project dealing with several inspection scenarios of power plants and resulting in many robot prototypes and 3 main achievements. The project is a common effort between industry and university, including ALSTOM together with researchers from the 2 Swiss Federal Institutes of Technology. The goal is to generate knowledge and transfer technology to the industry in the field of robot inspection. The method is to collect commercial scenarios, study them, make some exploratory prototypes, select the best scenarios and develop enhanced prototypes for the most promising applications. The 3 most evolved robots are MagneBike for steam chest, AirGapCrawler for generators and TubeCrawler for boiler tube. The supporting technologies that have been developed are adhesion, locomotion, system integration and localization.
\end{abstract}

Index Terms - industrial power system maintenance, inspection, mobile robots, Robot sensing systems.

\section{INTRODUCTION AND GOAL}

$\mathrm{I}_{\mathrm{o}}^{\mathrm{s}}$ NSPECTION of power plants is essential for a safe and optimal operation of the facilities. Plants get older and older and security assessments are both necessary and regulated by law. More and more robots are used to execute inspection because of several reasons: automatic or semiautomatic tasks, faster execution, enhanced precision, zones difficult to access, fast global assessment, or just to keep an exact history of the same measured position. This business is quite valuable but requires specific knowledge, adapted tools and experienced people.

This paper reports on a large applied research project started in 2007 dealing with several inspection scenarios of power plants and resulting in many prototypes and achievements. The project is a common effort between industry and university, including ALSTOM together with researchers from the two Swiss Federal Institutes of Technology in Zurich (ETHZ) and Lausanne (EPFL). The goal is to generate knowledge and transfer technology to the

This work was done in the frame of the Swiss CTI project 8435.1 "Highly compact robots for power plants inspections" supported by the Swiss government and ASLTOM.

G. Caprari, A. Breitenmoser, W. Fischer, C. Hürzeler, F. Tâche, R. Siegwart are with the Autonomous System Lab, ETH Zürich, Tannenstrasse 3, 8092 Zürich, Switzerland (e-mail: g.caprari@ieee.org).

P. Schoeneich, F. Rochat, F. Mondada are with the Laboratoire de Systèmes Robotiques (LSRO), Ecole Polytechnique Fédérale de Lausanne (EPFL), Switzerland (mobots.epfl.ch).

R. Moser is with Inspection Technologies, ALSTOM Power, Baden, Switzerland. industry. The method is to collect commercial scenarios, study them, make some exploratory prototypes, select the best scenarios and develop enhanced prototypes for the most promising applications. The selection criteria are business relevance, technical feasibility and complexity. Problems that can be solved with current State-of-the-Art are forwarded directly to industrial partners. Subjects requiring too much development and out of the scope of the timeframe will be continued in following research projects. The remaining complex but feasible projects are tackled within the current project. Fig. 1 shows the active scenarios and the milestones on the time axis per each researched field.

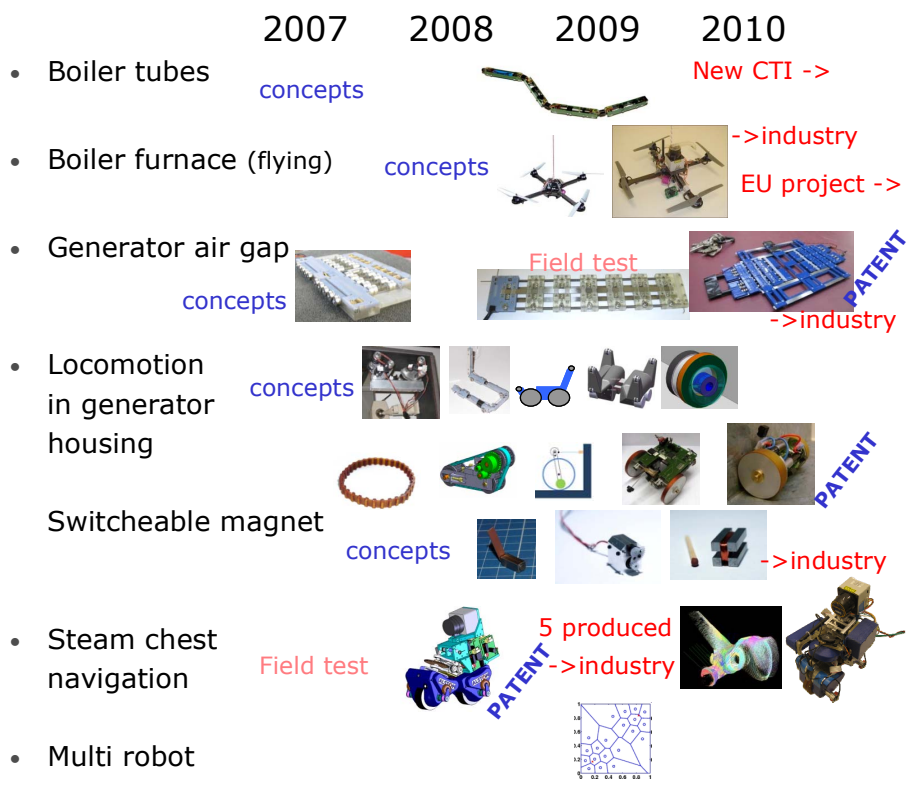

Fig. 1. Timescale and milestones of the global project. Start are the commercial needs, concept are realized and then prototypes are built and tested before going to an industrial phase or before stating a specific following-up research project.

The objective is to develop new robots and methods to be used in real scenarios of power plants to inspect areas like boilers, steam chests, generators and turbines. After a conceptual phase and some preliminary prototypes, field tests are executed with the best models. Developed robots and tangible results are for example the MagneBike [1] for steam chests, the AirGapCrawler [16] for generators or the TubeCrawler [15] for boiler tubes. Other results of the project are the created knowledge in adhesion, locomotion, localization and system integration used both in the final robots as well in many inspiring prototypes for future 
applications. Some long term research has started and will be continued. Inspection with flying robots for instance is the subject of the following-up European project AIRobots [20]. Research toward inspection in tubes will be continued with the goal of more robust and water resistant systems.

This paper is an overview on the full 3 years project and presents its main results. A common learned lesson as well as a common conclusion is also derived. More detailed results are referenced and are the object of specific scientific publications. The next chapter II discusses the necessary technology that has been developed as basis for most of the following prototypes. Chapter III presents the systems resulting from the study and finally chapter IV gives an outlook and a conclusion.

\section{TECHNOLOGY AND SCIENCE}

Inspection robots are mostly climbing robots that have to travel to specific points of the plant carrying the sensor to do measures. A necessary step toward the goal was to build knowledge in science and technology common to different scenarios and in the underlying research fields of climbing robots. Those are adhesion, locomotion, localization and system integration.

\section{A. Adhesion}

Inspecting structures means actually inspecting the surfaces composing the different parts, and thus being in close and safe contact with the surface is crucial in any direction regarding gravity. Adhesion can be achieved in many ways but being most of the industrial parts ferromagnetic, magnetisms is used extensively with either magnetic wheels or magnets in the body structure as in the presented prototypes. Magnetic wheels have the advantages to be in direct and close contact to the ferromagnetic underground and thus having a reduced air gap essential for strong adhesion. In addition they have a continuous smooth movement often necessary for the carried sensors. Magnets in the structure body are mechanically simpler and can be a choice when the payload of the vehicle is not too high. Moreover the latter suffer less from magnetic dust particles present in the environment and that get attached. Magnetic wheels can be optimized [7] to a specific vehicle to achieve very high adhesion as it is the case for the MagneBike robot. A special case is the wheel of Cy-mag3D [8] having the magnetic adhesion only in a section of the wheel that thus automatically redirect the robot toward the underlying surface and ease passing steps and obstacles. This concept generated a patent [26].

Magnets tend to get attracted anywhere in the environment and one might need to turn off the adhesion force. The technique of Magnetic Switchable Device (MSD) has many applications as presented in [6].

\section{B. Locomotion}

Even if new stronger magnets can generate a good adhesion, once those are to be put on vehicles with high mobility, smart new solutions have to be found. Especially in order to travel in complex environment and pass steps, corners, edges, gaps or difficult sequences of obstacles where the magnets tend to stick everywhere blocking the movement. Typically in a $90^{\circ}$ concave corner a magnetic wheel get blocked as it get attracted by both surfaces of the angle. Several solutions have been implemented in our global project in different robots:

- A lifter concentric with the magnetic wheel is used in MagneBike enabling to pass concave corners and sequences of steps. In [1][2][3] the concept is described and the high mobility achieved is pointed out. This is patented in [25].

- An adapted shape of the robot structure can also enable to efficiently and simply pass concave corners with just some extra wheels placed at the right position. A geometrical description is well discussed in [10].

- Also using the shape of the robot but with caterpillar including magnets is presented in [11].

- Non rounded wheels in the form of hexagons have been proven in [14] to be well suited to very small vehicle and to simply pass most obstacles.

- Even well tailored four-wheel-drive robot with normal magnetic wheels can pass concave corners. The geometry and the limitations on the necessary friction coefficient are analytically and experimentally can be found in [9].

Summarizing, there are various method how to use magnetic adhesion but still be able to pass obstacles. The best solution depends on the robot size and allowed complexity, the environment, the payload, the controllability and other application specific constraints. This is the subject of one of the thesis done during the whole project [24].

Another locomotion challenge in inspection is the limited entrance spaces like in turbines with their boroscope holes. [12] and [13] disclose an innovative concept based on changing totally the shape of the robot in order to pass trough small entrance holes and then fold from a tube-like shape in a full vehicle able to travel on bigger surfaces.

\section{1) Alternative Locomotion: Flying}

Instead of moving a robot on the surface to inspect and being blocked by the obstacles or having difficult to adhere to the surface, a totally alternative of locomotion is flying. Flying robots have become popular as the technology (light but powerful motors and batteries) is nowadays available so that small helicopters can fly during a reasonable time and lift some payload. An application scenario is having a teleoperated helicopter [18] flying in a boiler and approaching the boiler water wall to do visual inspection. Going further, one can imagine that the robot gets in contact with the wall and docks magnetically [19] to do a non destructive testing (NDT) with ultrasound. Till now the concept, the platform and the basic control are well advanced. The development continues with the specific European project AIRobots [20] that just started beginning 2010.

\section{Localization}

It is important to localize the vehicle doing the inspection within the plant or the component and thus know the place where the inspection measure is carried out. In simple cases a precise odometry based only on the wheel encoders is sufficient. In other cases, camera images can lead to the reconstruction of the path traversed with vision algorithms. 
But in non trivial environments, specific sensors have to be included to get a more robust localization. In the most advanced vehicle we included a 3D laser scanner [4] delivering high resolution 3D point clouds of the environment enabling an effective fusion with odometry to get a precise localization in complex environment like a steam chest [5]. This complex localization is surely inspired by state-of-the-art localization algorithms for robots evolving on planar or almost planar surfaces. However inspection robots move in full 3D environment with any orientation possible. Moreover the industrial environments to be inspected by robots are often complex, small, without distinctive features and without lighting. A chapter of the thesis [23] done within the project discusses and solves the issue in an elegant way using the MagneBike robot and the 3D scanner.

\section{System Integration}

System integration is the other common denominator of inspecting robots, required not only to make the system compact but also to get on board all the required functionalities. Mechanics for the mobility has to leave enough space for the controlling electronics and for the sensors. Sensors are both for ensuring the functionality of the robot (encoders, localization, ..) but also sensors for the final task that is inspection with cameras or with non destructive testing (NDT) equipment. In that sense the MagneBike is fully integrated having apart all the mechanics and motorization also the proprioceptive sensors and exteroceptive sensors like the laser scanner. Other examples are the Cy-mag3D [8] and the Tripillar [11] which have motors, mechanics, control, communication and also energy embedded.

\section{SYSTEMS}

The robots presented in this work (MagneBike, AirGapCrawler and TubeCrawler) are the most promising systems of the entire research project, and have reached a maturity that enable them to be soon industrialized.

\section{A. MagneBike}

The MagneBike (Fig. 2) is a robot in a bicycle configuration with 2 special magnetic wheels enhanced with lifter to get out of stocked situations in corners but also helpful as stabilizers on sideway paths. This configuration is suited to traverse junctions of tubes of variable diameters with steps in between and other obstacles that can be found in steam chest or other similar environment in power plants. The system is highly integrated with all the electronics onboard and even the option of a 3D scanner on top. Being well advanced, further research is on-going with the goal to use multiple units of those robots to speed up and make more robust the inspection process [21]. The challenge for multi robot inspection is to efficiently distribute the workload and thus the surface to inspect having a full coverage of the entire zone [22].

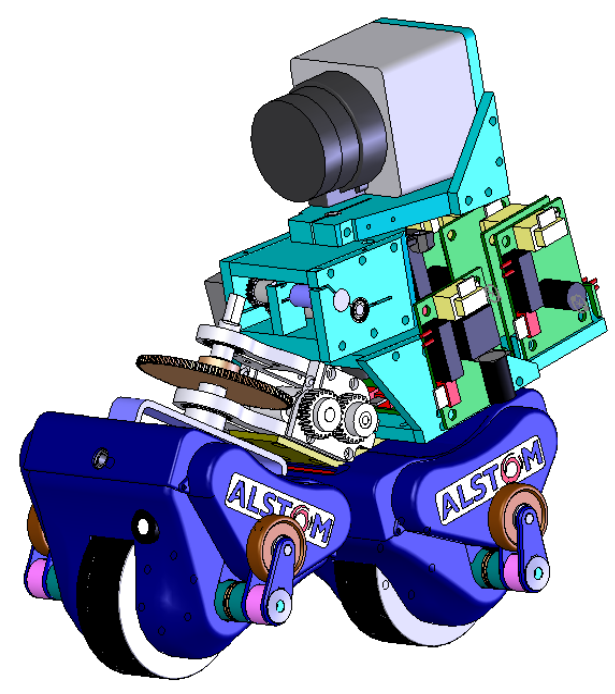

Fig. 2. MagneBike robot with 2 magnetic wheels with lifter/stabilizers on each wheel. Top there is a $2 \mathrm{D}$ laser scanner with an additional rotational to get a full 3D scan around the robot.

\section{B. AirGapCrawler}

The AirGapCrawler is a very thin robot $(8 \mathrm{~mm})$ that can travel between rotor and stator of electrical generators with the huge advantage in respect with the past to be small enough to be inserted without the need of removing the rotor from the generator. This is a critical and very time consuming task that is nowadays often unavoidable as no general robotic tools exist. Reasons are both the small gap between rotor and stator but also because of the complex shape of the entrance. We present here a couple of versions with different mobility but with the same core being a very compact set of magnetic wheels and alignment mechanisms. In Fig. 3 you can see the 20 central magnetic wheels generating the adhesion and on the left side the 10 wheels that can be folded down 90' to get aligned on the stator structure. On the right side of the Fig. 3 there is a schema of the generator air gap where the thin robot should enter and move. The first version travels in the gap only axially [16], whereas the second one is also able to move circumferentially with an inchworm sequence [17]. The second movement is used to avoid obstacles in some generators or to pass automatically from one line of tooth to the next.

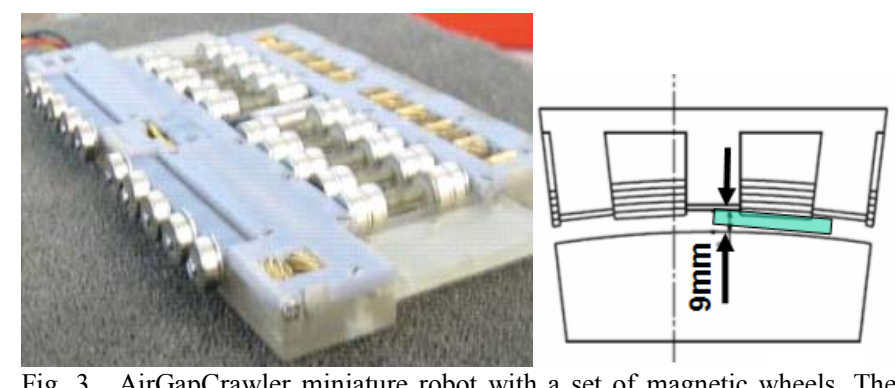

Fig. 3. AirGapCrawler miniature robot with a set of magnetic wheels. The robot is only $8 \mathrm{~mm}$ thin to pass in the air gap between rotor and stator of big generators. 


\section{TubeCrawler}

The TubeCrawler (Fig. 4), also called Tubulo [15], is a robot developed to inspect the tubes around the furnace of a boiler where the water is warmed up, the so called water wall. The typical internal diameter of the tubes measures 1 inch and the length is around 25 meters. However there might be diameter changes and there are bends of 6 inches of radius. Because of the diameter changes, magnetic adhesion has been chosen and the robot has magnetic wheels. A train like structure with 4 wagons has been selected to be mechanically flexible both but also to have the possibility to use different configurations of sensors. The connectors between the modules have a magnet to exchange rapidly the modules. The images from the camera in front are transmitted via the cable at the end of the train toward a user control station.

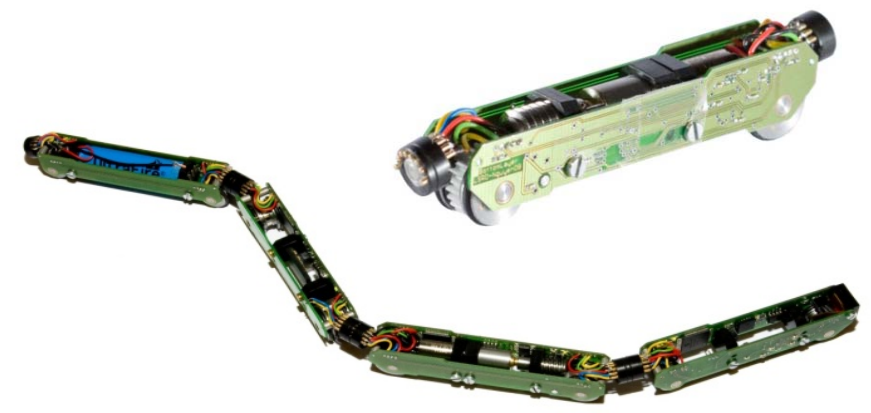

Fig. 4. TubeCrawler can drive in 1 inch tubes of boiler water walls. The train configuration has a module for motor, battery, camera and control and communication.

\section{OUTLOOK AND CONCLUSION}

Future developments include the enhancement of the existing systems to be more robust against hazards and dirt, more integrated and more autonomous. For instance the MagneBike robot will be partially autonomous in negotiating steps, driving autonomously the lateral lifter/stabilizers without constantly requiring user interventions. The AirGapCrawler will be more flexible, able to adapt to uneven surfaces that can be found in some generators. The TubeCrawler will be more enclosed, screened against dust.

Another direction with a complete different paradigm is towards flying systems for fast visual inspection where available space is less of an issue. We can expect revolutionary results when the flying system, their control and their integration will be further developed.

Looking back to the 3 years project and seeing the results of such an applied research project, we can be confident that many new ideas and methods can be used very soon in industrial inspection of power plants. The systems built in the project are directly useful for real inspection tasks. The basic technology and science developed during the 3 years can be rapidly exploited for other inspection scenarios. In particular the magnetic adhesion, the locomotion concepts and the 3D localization will be common to any other robot within the same kind of environment. Moreover, the developed basic knowledge will lead to new solutions in this or nearby application fields.

\section{REFERENCES}

*** MagneBike + Navigation

[1] F. Tâche, W. Fischer, R. Moser, F. Mondada, R. Siegwart, "Adapted Magnetic Wheel Unit for Compact Robots Inspecting Complex Shaped Pipe Structures", Proc. of The IEEE/ASME International Conference on Advanced Intelligent Mechatronics (AIM), 2007.

[2] F. Tâche, W. Fischer, R. Siegwart, R. Moser, F. Mondada, "Compact Magnetic Wheeled Robot With High Mobility for Inspecting Complex Shaped Pipe Structures", Proc. of The IEEE/RSJ International Conference on Intelligent Robots and Systems (IROS), 2007.

[3] F. Tâche, W. Fischer, R. Moser, F. Mondada, R. Siegwart, "Magnebike: A Magnetic Wheeled Robot With High Mobility for Inspecting Complex Shaped Structures", Journal of Field Robotics, 2009.

[4] L. Kneip, F. Tache, C. Caprari, R. Siegwart, "Characterization of the compact Hokuyo URG-04LX 2D laser range scanner", ICRA09, 2009.

[5] F. Tâche, F. Pomerleau, W. Fischer, G. Caprari, F. Mondada, R. Moser, R. Siegwart, "MagneBike: Compact Magnetic Wheeled Robot for Power Plant Inspection", CARPI 2010, Video Submission

*** Magnetic adhesion

[6] F. Rochat, P. Schoeneich, M. Bonani, , S. Magnenat, C. Hürzeler, F. Mondada, H. Blueler, "Design of Magnetic Switchable Device (MSD) and applications in climbing robot", Proc. of The 13th International Conference on Climbing and Walking Robots and the Support Technologies for Mobile Machines (CLAWAR), September 2010.

[7] F. Rochat, P. Schoeneich, F. Tâche, F. Mondada, H. Blueler, "Magnetic wheels optimization and application to the MagneBike climbing robot", Proc. of The 13th International Conference on Climbing and Walking Robots and the Support Technologies for Mobile Machines (CLAWAR), September 2010.

[8] F. Rochat, P. Schoeneich, B. Luthi, F. Mondada, H. Blueler, "Cymag3D: a simple and miniature climbing robot with advance mobility in ferromagnetic environment", Proc. of The 13th International Conference on Climbing and Walking Robots and the Support Technologies for Mobile Machines (CLAWAR), September 2010.

*** Locomotion

[9] W. Fischer, F. Tâche, G. Caprari, R. Siegwart, "Magnetic Wheeled Robot with High Mobility but only 2 DOF to Control", Proc. of The 11th International Conference on Climbing and Walking Robots and the Support Technologies for Mobile Machines (CLAWAR), 2008.

[10] W. Fischer, G. Caprari, R. Siegwart, R. Moser, "Compact Magnetic Wheeled Robot for Inspecting Complex Shaped Structures in Generator Housings and Similar Environments", Proc. of the 2009 IEEE/RSJ International Conference on Intelligent Robots and Systems, 2009.

[11] F. Rochat, P. Schoeneich, O. Truong-Dat Nguyen, F. Mondada "TRIPILLAR: miniature magnetic caterpillar climbing robot with plane transtion ability", CLAWAR09, 2009.

[12] W. Fischer, G. Caprari, R. Siegwart, R. Moser, "Foldable Magnetic Wheeled Climbing Robot for the Inspection of Gas Turbines", Proc. of The 12th International Conference on Climbing and Walking Robots and the Support Technologies for Mobile Machines (CLAWAR), Winner of the Industrial-Robot Innovation award 2009

[13] W. Fischer, G. Caprari, R. Siegwart, I. Thommen, W. Zesch, R. Moser, "Foldable Magnetic Wheeled Climbing Robot for the Inspection of Gas Turbines and Similar Environments with very Narrow Access Holes", Industrial Robot: An International Journal, Vol. 37, No. 3, May 2010, 244-249.

[14] W. Fischer, G. Caprari, R. Siegwart, R. Moser, "Very Compact Climbing Robot rolling on Magnetic Hexagonal Cam-Discs, with High Mobility on Obstacles but Minimal Mechanical Complexity", ISR / ROBOTIK 2010,

[15] P. Schoeneich, F. Rochat, O. Truong Nguyen, G. Caprari, R. Moser, H. Bleuler, F. Mondada, "Tubulo - a train-like miniature inspection climbing robot for ferromagnetic tubes", CARPI 2010.

*** AirGapCrawler

[16] W. Fischer, G. Caprari, R. Siegwart, R. Moser, "Robotic Crawler for Inspecting Generators with Very Narrow Air Gaps", Proc. of The 5th IEEE International Conference on Mechatronics (ICM), 2009.

[17] W. Fischer, G. Caprari, R. Siegwart, R. Moser, "Robotic crawler at very low height with axial and circumferential mobility - for inspecting generators with the rotor still installed", Transaction on Industrial Electronics, 2010 
*** Flying

[18] C. Hürzeler et al, "Teleoperation Assistance for an Indoor Quadrotor Helicopter", Proceedings of International Conference on Simulation, Modeling and Programming for Autonomous Robots (SIMPAR),2008

[19] W. Fischer, C. Hürzeler, R. Siegwart, "Lightweight magnetic foot for docking unmanned helicopters to steel walls", Proc. of The 13h International Conference on Climbing and Walking Robots and the Support Technologies for Mobile Machines (CLAWAR), 2010.

[20] AIRobots: "Innovative aerial service robots for remote inspections by contact", European research project, www.airobots.eu. 2010-2013

*** Multi robot

[21] A. Breitenmoser, F. Tâche, G. Caprari, R. Siegwart, R. Moser, "MagneBike - Toward Multi Climbing Robots for Power Plant Inspection", Proc. of the 9th International Conference on Autonomous Agents and Multiagent Systems (AAMAS), May 2010.

[22] A. Breitenmoser, M. Schwager, J. Metzger, R. Siegwart, D. Rus, "Voronoi Coverage of Non-Convex Environments with a Group of Networked Robots", Proc. of the IEEE International Conference on Robotics and Automation (ICRA), May 2010.

Dissertations:

[23] F. Tâche, "Robot Locomotion and Localization on 3D Complex Shaped Structures", Ph.D. dissertation, Dept. of Mechanical and Process Eng., ETH Zurich, Switzerland, 2010.

[24] W. Fischer, "Design of robots for power plant inspection", Ph.D dissertation, Dept. of Mechanical and Process Eng., ETH Zurich, Switzerland, 2010

Patents:

[25] R. Moser, W. Fischer, F. Tache, R. Siegwart, F. Mondada, "Automotive inspection vehicle", EP 2003044 A1, 14.06.2007

[26] R. Moser, F. Mondada, P. Schoneich, F. Rochat, "Self-Stabilizing one Axis Inspection Robot with Magnetic Wheels", Swiss Patent, 27.11.2009.

[27] W. Fischer, M. Wiesendanger, "Device for Inspection of Generator Air Gaps", US patent, 19.1.2010.

[28] W. Fischer, "Robotic Inspection Vehicle with High Mobility in Difficult Transitions at Minimum Mechanical and Control Complexity", Swiss Patent, 14.4.2010.

\section{BIOGRAPHIES}

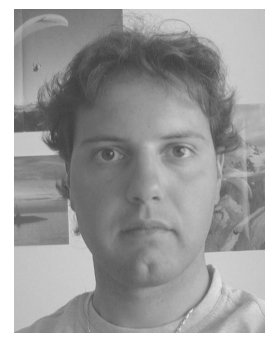

Gilles Caprari received his M.Sc. in Electrotechnical engineering in 1996 at ETH Zurich and his PhD in 2003 at EPFL Lausanne. He has been research assistant at ETH Zurich and EPFL. He founded GCtronic (2005, Development and consulting, Production, sale and support of mobile robots) and Skybotix (2009, Innovative Micro Aerial Vehicles). His interests include robot design, miniature robots, robotics applications, flying robots, educational robotics and application oriented research. He was project leader of the CTI applied research project on Inspection Robots for Power Plants (2006-2010). Hi is now involved with the EU project AIRobots.

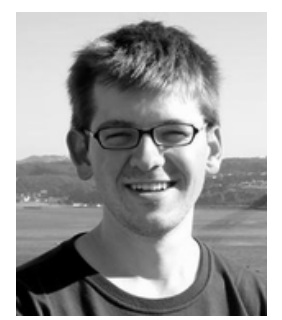

Andreas Breitenmoser received his M.Sc in Electrotechnical Engineering from ETH Zurich in 2008. He is currently $\mathrm{PhD}$ student at the Autonomous Systems Lab of ETH Zurich. His main topic is multi robot control and coverage for inspection tasks. $\mathrm{He}$ is cooperating with $\mathrm{CMU}$ CSAIL Lab for robot distributed control.

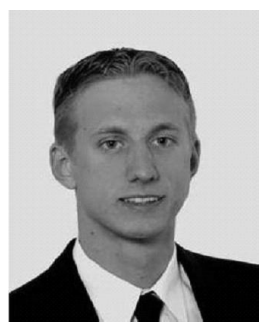

Wolfgang Fischer received the academic degrees "Diplom-Ingenieur Univ." in mechanical engineering from TU München, Germany and "Ingeniero Industrial" from Universidad Politécnica de Madrid, Spain in 2005 and the Ph.D. degree in robotics from ETH Zürich, Switzerland in 2010. He currently works at the Autonomous Systems Lab in ETH Zürich as a Post-Doc and for ALSTOM Inspection Robotics as a research engineer. His main interests are the development of innovative locomotion principles for enhancing the mobility of compact mobile robots and the system-integration and industrialization of such systems - with main focus on compact robots with magnetic adhesion and their applications in inspection tasks in the power industry.

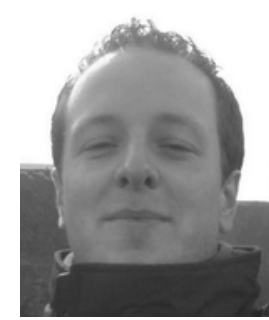

Christoph Hürzeler received his M.Sc in Mechanical Engineering from ETH Zurich in 2007. $\mathrm{He}$ is currently $\mathrm{PhD}$ student at the Autonomous Systems Lab of ETH Zurich. His main topic is flying platform for inspection tasks with particular interest in control, design and docking. He participated to the CTI project Highly Compact Robots for Inspection of Power Plants and he is now involved with the EU project AIRobots.

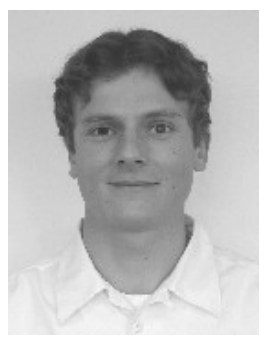

Fabien Tâche received his M.Sc. in MicroEngineering in 2004 from the Ecole Polytechnique Fédérale de Lausanne (EPFL). He carried out his master thesis at the Robotics Institute from Carnegie Mellon University under the supervision of Prof. Illah Nourbakhsh. In June 2004, he started working on the Leurre project at the Autonomous Systems Laboratory (ASL) of Prof. R. Siegwart. At the end of 2005 , he started his doctoral thesis in the same laboratory in the field of design and control of climbing inspection robots. In 2006, he moved with Prof. R. Siegwart to the ETH Zurich where he received the Doctoral Degree in 2010. His main interests are climbing robots for inspection of narrow environments, miniature robots, and mechatronics systems.

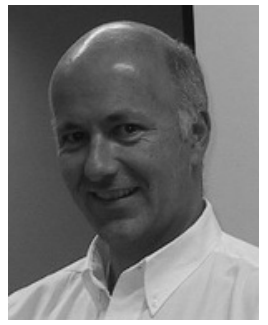

Roland Siegwart is vice president of ETH Zürich since 2009 and full professor for autonomous systems at ETH Zürich since July 2006. He has a Diploma in Mechanical Engineering (1983) and PhD in Mechatronics (1989) from ETH Zurich. In $1989 / 90$ he spent one year as postdoctoral fellow at Stanford University. After that he worked part time as R\&D director at MECOS Traxler AG and as lecturer and deputy head at the Institute of Robotics, ETH Zürich. In 1996 he was appointed as associate and later full professor for autonomous microsystems and robots at the Ecole Polytechnique Fédérale de Lausanne (EPFL). During this period at EPFL he was co-initiator and founding Chairman of Space Center EPFL and Vice Dean of the School of Engineering. In 2005 he held visiting positions at NASA Ames and Stanford University. Roland Siegwart is member of the Swiss Academy of Engineering Sciences, IEEE Fellow and board member of the European Network of Robotics (EURON). He served as Vice President for Technical Activities (2004/05) and Distinguished Lecturer (2006/07) and is currently AdCom Member (2007-2009) of the IEEE Robotics and Automation Society. He leads a research group of around 35 people working in the field of robotics, mechatronics and product design. He is coordinator of two large European projects and co-founder of several spin-off companies. 


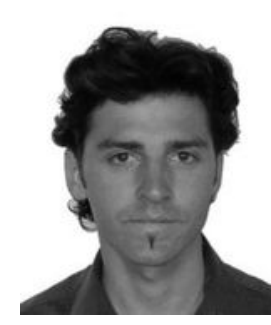

Patrick Schoeneich obtained a Master in Microengineering at Ecole Polytechnique Fédérale de Lausanne (EPFL) in March 2007. He is working on his $\mathrm{PhD}$ in robotics at LSRO (EPFL), concentrating on the system integration of miniature inspection robots.



Frédéric Rochat received his Master in Microengineering at Ecole Polytechnique Fédérale de Lausanne (EPFL) in March 2007. He is working on his $\mathrm{PhD}$ in robotics at LSRO (EPFL), concentrating on the system integration of miniature inspection robots.

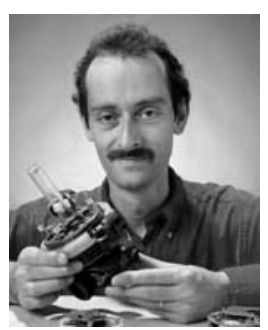

Francesco Mondada received his M.Sc. in microengineering in 1991 and his Doctoral degree in 1997 at EPFL. During his thesis he co-founded the company K-Team, being both CEO and president of the company for about 5 years. He is one of the three main developers of the Khepera robot, considered as a standard in bio-inspired robotics and used by more than 1,000 universities and research centers worldwide. He participated to the SWARMBOTS project as the main developer of the s-bot robot platform. In November 2005 he received the EPFL Latsis University prize for his contributions to bio-inspired robotics. His interests include the development of innovative mechatronic solutions for mobile and modular robots, the creation of know-how for future embedded applications, and making robot platforms more accessible for education, research, and industrial development.

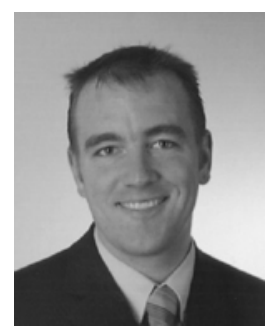

Roland Moser received a M.Sc. in Precision Engineering, micro technologies in ETHZ, and his $\mathrm{PhD}$ at EPFL in 2002. He has been Manager of the Electronic Department of SSM AG (2003-2005) and Director of R\&D Inspection Technologies at ALSTOM Power (2005-2010). He is now the Department Head of R\&D Inspection Methods and Robotics/Automation at Alstom Power. 